Journal of Mathematical Physics, Analysis, Geometry

2014, vol. 10, No. 4, pp. 430-450

\title{
Functional Models in De Branges Spaces of One Class Commutative Operators
}

\author{
V.N. Syrovatskyi \\ V.N. Karazin Kharkiv National University \\ 4 Svobody Sq., Kharkiv 61077, Ukraine \\ E-mail: VSirovatsky@gmail.com
}

Received March 17, 2013, revised May 26, 2014

\begin{abstract}
For a commutative system of the linear bounded operators $T_{1}, T_{2}$, which operate in the Hilbert space $H$ and none of the operators $T_{1}, T_{2}$ is a compression, the functional model is constructed. The model is built for a circle in de Branges space.

Key words: functional model, de Branges space, commutative systems of operators.
\end{abstract}

Mathematics Subject Classification 2010: 47B32.

The functional model of the compression operator $T$ acting in the Hilbert space $H$ was first obtained by B.S. Nagy and C. Foias [5]. The model allows to present the operator $T$ as an operator of multiplication by the independent variable in a special space of functions [5,2]. The study of the spectral characteristics of this model has led to a number of non-trivial problems on either functional analysis or theory of functions including the issues of interpolation, tasks of basis, completeness, etc. [2].

When the Nagy-Foias dilation technique [5] was used, there appeared significant difficulties in the constructing of similar functional models for the commutative systems of the operators $\left\{T_{1}, T_{2}\right\}$ defined in the Hilbert space $H$. Thus, the above problem could not be solved even for $T_{1}$ and $T_{2}$ being compressible. The solution was found in [7], which is based on a generalization of the concept node for commutative system operators, and in fact was proposed by Livshits.

In [8], a functional model of a pair of commutative operators is built when one of them is compressed. The construction is based on the Fourier transformation technique. If none of the operators $\left\{T_{1}, T_{2}\right\}$ is not a compression, then the given method is not applicable. In this paper, we construct the functional models for a commutative system of the operators $\left\{T_{1}, T_{2}\right\}$ where neither $T_{1}$ nor $T_{2}$ is compressed. For this case the functional model is constructed in de Branges space corresponding to the unit circumference obtained in [6].

(c) V.N. Syrovatskyi, 2014 


\section{Background Information}

Let us consider the bounded linear operator $T$ acting in the Hilbert space $H$. The collection

$$
\triangle=\left(J ; H \oplus E ; V=\left[\begin{array}{cc}
T & \Phi \\
\Psi & K
\end{array}\right] ; H \oplus \tilde{E} ; \tilde{J}\right)
$$

is called a unitary knot [1-4] if the linear operator

$$
V=\left[\begin{array}{ll}
T & \Phi \\
\Psi & K
\end{array}\right]: H \oplus E \mapsto H \oplus \tilde{E}
$$

satisfies the correlation

$$
V^{*}\left[\begin{array}{cc}
I & 0 \\
0 & \tilde{J}
\end{array}\right] V=\left[\begin{array}{ll}
I & 0 \\
0 & J
\end{array}\right], \quad V\left[\begin{array}{ll}
I & 0 \\
0 & J
\end{array}\right] V^{*}=\left[\begin{array}{cc}
I & 0 \\
0 & \tilde{J}
\end{array}\right],
$$

where $J$ and $\tilde{J}$ are involutions in the Hilbert spaces $E$ and $\tilde{E}$, respectively, $J=$ $J^{*}=J^{-1}, \tilde{J}=\tilde{J}^{*}=\tilde{J}^{-1}$. Any bounded linear operator $T$ in $H$ can always be included into a unitary knot $\triangle(1.1)$ if we set $[2],-E=\overline{D_{T^{*}} H} ; \tilde{E}=\overline{D_{T} H} ; \Psi=$ $\sqrt{\left|D_{T}\right|} ; \Phi=\sqrt{\left|D_{T^{*}}\right|} ; J=\operatorname{sign} D_{T^{*}} ; \tilde{J}=\operatorname{sign} D_{T} ; K=-\tilde{J} T^{*} ;$ where, as usually, $D_{T}=I-T^{*} T$ are defective operators of $T$, and $\sqrt{|A|}, \operatorname{sign} A$ of the self-adjoint operator $A$ are understood in terms of the corresponding spectral decompositions.

The knot $\triangle(1.1)$ is called simple [2] if $H=H_{1}$, where

$$
H_{1}=\operatorname{span}\left\{T^{n} \Phi f+T^{* m} \Psi^{*} g ; f \in E ; g \in \tilde{E} ; n, m \in \mathbb{Z}_{+}\right\} .
$$

The subspaces $H_{1}$ and $H_{0}=H_{1}^{\perp}=H \ominus H_{1}$ reduce the operator $T$, and the reducing of $T$ to $H_{0}$ is a unitary operator [2].

The main invariant of the knot $\triangle(1)$, which describes simple knots, is a characteristic operator function introduced by Livshits in 1946, [1],

$$
S_{\triangle}=K+\Psi(z I-T)^{-1} \Phi,
$$

which plays the main role in the theory of triangular [2] and functional models $[4,5]$ for the operators close to the unitary ones (in terms of definition (1.1)).

Suppose that $\operatorname{dim} E=\operatorname{dim} \tilde{E}=r<\infty$ and $J=\tilde{J}$. Let us choose the orthonormalized bases $\left\{e_{\alpha}\right\}_{1}^{r}$ and $\left\{e_{\alpha}^{\prime}\right\}_{1}^{r}$ in $E$ and $\tilde{E}$. Then from the results of Potapov [2] it follows that the matrix-function $S_{\triangle}(z)=\|\left\langle S_{\triangle}(z) e_{\alpha}, e_{\beta}^{\prime}\right\rangle$ $\|$, in the case when the spectrum $\sigma(T)$ of the operator $T$ belongs to the unit circumference $\mathbb{T}=\{z \in \mathbb{C} ;|z|=1\}$, has the multiplicative structure

$$
S_{\triangle}(z)=\int_{0}^{\overleftarrow{l}} \exp \left\{\frac{e^{\imath \varphi_{t}}+z}{e^{\imath \varphi_{t}}-z} J d F_{t}\right\},
$$


where $\varphi_{t}$ is a non-negative non-decreasing on $[0, \ell]$ function, and $0 \leq \varphi_{t} \leq 2 \pi ; F_{t}$ is a non-decreasing hermitian $(r \times r)$ matrix-function on $[0, \ell]$ for which $\operatorname{tr} F_{t} \equiv t$.

Using Potapov's presentation (1.6), it is not difficult to build a triangular model of the operator for $S_{\triangle}(z)(5)$. By $L_{r, l}^{2}\left(F_{x}\right)$, denote the Hilbert space of the vector functions

$$
L_{r, l}^{2}\left(F_{x}\right)=\left\{f(x)=\left(f_{1}(x), \ldots, f_{r}(x)\right) ; \int_{0}^{l} f(x) d F_{x} f^{*}(x)<\infty\right\} .
$$

In $L_{r, l}^{2}\left(F_{x}\right)(1.7)$, define the linear operator $T$,

$$
T f(x)=f(x) e^{\imath \varphi_{x}}-2 \int_{x}^{l} f(t) d F_{t} \Phi_{t}^{*} \Phi_{x}^{*-1} J e^{\imath \varphi_{x}},
$$

where the matrix $\Phi_{x}$ is a solution of the integral equation

$$
\Phi_{x}+\int_{0}^{x} \Phi_{t} d F_{t} J=I, \quad x \in[0, l] .
$$

Similarly, the matrix-function $\Psi_{x}$ is a solution of

$$
\Psi_{x}+\int_{x}^{l} \Psi_{t} d F_{t} J=J, \quad x \in[0, l] .
$$

Let us define the operators $\Phi: E \mapsto L_{r, l}^{2}\left(F_{x}\right)$ and $\Psi: L_{r, l}^{2}\left(F_{x}\right) \mapsto E\left(\right.$ here $\left.E=\mathbb{C}^{n}\right)$ as follows:

$$
\Phi f(x)=\sqrt{2} f \Psi_{x} e^{\imath \varphi_{x}}, \quad \Psi f(x)=\sqrt{2} \int_{0}^{l} f(x) d F_{x} \Phi_{x}^{*},
$$

where $f \in E$ and $K=S_{\triangle}(\infty)$ (1.6). The collection

$$
\triangle_{c}=\left(J ; L_{r, l}^{2}\left(F_{x}\right) \oplus E ; V=\left[\begin{array}{cc}
T & \Phi \\
\Psi & K
\end{array}\right] ; L_{r, l}^{2}\left(F_{x}\right) \oplus E ; J\right)
$$

is a unitary knot (1.1)-(1.3) and is called a triangular model of the simple knot $\triangle$ (1.1), where $L_{r, l}^{2}\left(F_{x}\right), T, \Phi, \Psi$ are from $(1.7),(1.8)-(1.11)$. The latter means that simple components (1.4) of the knots $\triangle(1.1)$ and $\triangle_{c}$ (1.12), when the spectrum of the operator $T$ is on the unit circumference $\sigma(T) \subseteq \mathbb{T}$, are unitarily equivalent 
[2] under the condition that $J=\tilde{J}$ and $\operatorname{dim} E=\operatorname{dim} \tilde{E}=r<\infty$.

Let us suppose that $\operatorname{dim} E=2$ and $J=J_{N}$, where

$$
J_{N}=\left[\begin{array}{cc}
-1 & 0 \\
0 & 1
\end{array}\right]
$$

According to [6], we introduce

$$
\begin{gathered}
L_{x}(z)=(1-z T)^{-1} \Phi(1,1), \\
\widetilde{L}_{x}(z)=\left(1-z T^{*}\right)^{-1} \Psi^{*}(1,-1) .
\end{gathered}
$$

Definition 1. The de Branges space $\mathcal{B}(E, G)$ is a Hilbert space formed by the vector functions $F(z)=\left[F_{1}(z), F_{2}(z)\right]$, where $F_{k}(z),(k=1,2)$ are

$$
F_{1}(z)=\int_{0}^{l} f(t) d F_{t} L_{t}^{*}(\bar{z}), \quad F_{2}(z)=\int_{0}^{l} f(t) d F_{t} \tilde{L}_{t}^{*}(\bar{z}) .
$$

Present the de Branges space as follows:

$$
\mathcal{B}_{\phi} f=\left[F_{1}(z), F_{2}(z)\right]
$$

The scalar product in $\mathcal{B}(E, G)$ is induced by the prototype mapping $\mathcal{B}_{\varphi}(1.17)$,

$$
<F(z), \hat{F}(z)>_{\mathcal{B}_{\varphi}(E, G)}=<f(t), \hat{f}(t)>_{L_{2, l}^{2}\left(F_{t}\right)},
$$

while $F(z)=\mathcal{B}_{\varphi} f(t), \hat{F}(z)=\mathcal{B}_{\varphi} \hat{f}(t)$, where $f(t), \hat{f}(t) \in L_{2, l}^{2}\left(F_{t}\right)$.

The functions $E_{x}(z), \widetilde{E}_{x}(z), G_{x}(z), \widetilde{G}_{x}(z)$ are defined by the relations [6]

$$
\begin{gathered}
L_{x}(z)=\left(e^{-i \phi_{x}}-z\right)^{-1}\left[E_{x}(z), \widetilde{E}_{x}(z)\right], \\
\widetilde{L}_{x}(z)=\left(1-z e^{-i \phi_{x}}\right)^{-1}\left[G_{x}(z), \widetilde{G}_{x}(z)\right] .
\end{gathered}
$$

Let $T_{1}, T_{2}$ be a commutative system of the linear bounded operators acting in the Hilbert space $H$. The collection of the Hilbert spaces $E, \tilde{E}$ and the operators $\Phi \in$ $[E, H] ; \Psi \in[H, \tilde{E}] ; K \in[E, \tilde{E}] ; \sigma_{s}, \tau_{s}, N_{s}, \Gamma_{1} \in[E, E] ; \tilde{\sigma}_{s}, \tilde{\tau}_{s}, \tilde{N}_{s}, \tilde{\Gamma}_{1} \in[\tilde{E}, \tilde{E}](s=$ $1,2)$ is called a commutative unitary metric knot $\triangle$,

$$
\triangle=\left(\Gamma_{1}, \sigma_{s}, \tau_{s}, N_{s}, H \oplus E, V_{s}, \stackrel{+}{V}_{s}, H \oplus \tilde{E}, \tilde{N}_{s}, \tilde{\tau}_{s}, \tilde{\sigma}_{s}, \tilde{\Gamma}_{1}\right)
$$

if for the expansions

$$
V_{s}=\left[\begin{array}{cc}
T_{s} & \Phi N_{s} \\
\Psi & K
\end{array}\right], \quad \stackrel{+}{V}_{s}=\left[\begin{array}{cc}
T_{s}^{*} & \Psi^{*} \tilde{N}_{s}^{*} \\
\Phi^{*} & K^{*}
\end{array}\right]
$$


the following relations are true:

1) $V_{s}^{*}\left[\begin{array}{cc}I & 0 \\ 0 & \tilde{\sigma}\end{array}\right] V_{s}=\left[\begin{array}{cc}I & 0 \\ 0 & \tau_{s}\end{array}\right], \quad \stackrel{+}{V_{s}^{*}}\left[\begin{array}{cc}I & 0 \\ 0 & \sigma_{s}\end{array}\right] \stackrel{+}{V}_{s}=\left[\begin{array}{cc}I & 0 \\ 0 & \tilde{\tau}_{s}\end{array}\right]$,

2) $T_{2} \Phi N_{1}-T_{1} \Phi N_{2}=\Phi \Gamma_{1}, \tilde{N}_{1} \Psi T_{2}-\tilde{N}_{2} \Psi T_{1}=\tilde{\Gamma}_{1} \Psi$,

3) $\tilde{N}_{2} \Psi \Phi N_{1}-\tilde{N}_{1} \Psi \Phi N_{2}=K \Gamma_{1}-\tilde{\Gamma}_{1} K, K N_{s}=\tilde{N}_{s} K(s=1,2)$,

where $\sigma_{s}, \tau_{s},\left(\tilde{\sigma}_{s}, \tilde{\tau}_{s}\right)$ are self-adjoint in $E(\tilde{E}),(s=1,2)$.

The operators acting in the spaces $E$ and $\tilde{E}$ of the knot $\triangle(1.21)$ are dependent. An arbitrary commutative system of the linear bounded operators $T_{1}, T_{2}$ can always be included into the knot $\triangle(1.21)$ [1]. If the "defective" operators $\sigma_{1}$ and $\tilde{\sigma}_{1}$ in $E$ and $\tilde{E}$ are reversible, we can always suppose that $N_{1}$ and $\tilde{N}_{1}$ are reversible. Let us introduce $N, \tilde{N}, \Gamma, \tilde{\Gamma}$ in the following form:

$$
N=N_{1}^{-1} N_{2}, \quad \Gamma=N_{1}^{-1} \Gamma_{1}, \quad \tilde{N}=\tilde{N}_{1}^{-1} \tilde{N}_{2}, \quad \tilde{\Gamma}=\tilde{N}_{1}^{-1} \tilde{\Gamma}_{1} .
$$

Let us set the linear operators $T_{1}$ and $T_{2}$ in $L_{r, l}^{2}\left(F_{x}\right)(1.7)$ :

$$
\begin{gathered}
T_{1} f(x)=f(x) e^{\imath \varphi_{x}}-2 \int_{x}^{l} f(t) d F_{t} \Phi_{t}^{*} \Phi_{x}^{*-1} J e^{\imath \varphi_{x}}, \\
T_{2} f(x)=f(x)\left(N(x) e^{\imath \varphi_{x}}+\Gamma(x)\right)-2 \int_{x}^{l} f(t) d F_{t} \Phi_{t}^{*} \Phi_{x}^{*-1} J N(x) e^{\imath \varphi_{x}},
\end{gathered}
$$

where $N(x)$ and $\Gamma(x)$ satisfy the differential Lax equations [7]:

$$
\begin{gathered}
N^{\prime}(x)=\left[a_{x} J, N(x)\right], \quad N(0)=\tilde{N}_{2}, \quad \Gamma^{\prime}(x)=\left[\Gamma(x), a_{x} J\right], \quad \Gamma(0)=\tilde{\Gamma}_{2}, \\
{\left[a_{x} J, \Gamma(x)+e^{\imath \varphi_{x}} N(x)\right]=0,}
\end{gathered}
$$

where $d F_{x}=a_{x} d x$.

\section{Effect of Operators $T_{1}$ and $T_{1}^{*}$ on Vectors $L_{x}$ and $\widetilde{L}_{x}$}

Let the knot $\triangle(1.21)$ corresponds to the commutative system of the operators $\left\{T_{1}, T_{2}\right\}$. Suppose that $E=\tilde{E}, \operatorname{dim} E=\operatorname{dim} \tilde{E}=2$ and $\sigma_{1}=\tilde{\sigma}_{1}=J_{N}$ (1.13), the spectrum of the operator $T_{1}$ consists of one point $\{1\}$, and therefore, $\varphi_{x}=0$. By $L_{x}(z)$ and $\tilde{L}_{x}(z)$, denote the vector functions (1.14),(1.15) which correspond to the operator $T_{1}\left(T=T_{1}\right)$. We also denote the functions $E_{x}(z), \widetilde{E}_{x}(z), G_{x}(z), \widetilde{G}_{x}(z)$ by $(1.19),(1.20)$.

Lemmas 1-4 were proved in [9]. They define the effect of the operators $T_{1}$ and $T_{1}^{*}$ on the vectors $L_{x}$ and $\widetilde{L}_{x}$. 
Lemma 1. [9] The operator $T_{1}$ affects the vector function $L_{x}(z)$ (1.14) in the following way:

$$
T_{1} L_{x}(z)=\frac{L_{x}(z)-L_{x}(0)}{z} .
$$

Lemma 2. [9] The operator $T_{1}$ affects the vector function $\widetilde{L}_{x}(z)$ (1.15) in the following way:

$$
T_{1} \widetilde{L}_{x}(z)=z \widetilde{L}_{x}(z)+\frac{\widetilde{G}_{l}(z)-G_{l}(z)}{2} L_{x}(0)-\frac{\widetilde{G}_{l}(z)+G_{l}(z)}{2}(1,-1) \Psi_{x} .
$$

Lemma 3. [9] The operator $T_{1}^{*}$ affects the vector function $\widetilde{L}_{x}(z)$ in the following way:

$$
T_{1}^{*} \widetilde{L}_{x}(z)=\frac{\widetilde{L}_{x}(z)-\widetilde{L}_{x}(0)}{z} .
$$

Lemma 4. [9] The operator $T_{1}^{*}$ affects the vector function $L_{x}(z)$ in the following way:

$$
T_{1}^{*} L_{x}(z)=z L_{x}(z)+\frac{E_{0}(z)-\widetilde{E}_{0}(z)}{2} \widetilde{L}_{x}(0)+\frac{E_{0}(z)+\widetilde{E}_{0}(z)}{2}(1,1) \Phi_{x} .
$$

Let us prove the lemma below.

Lemma 5. If the vector functions $L_{x}(z)$ and $\widetilde{L}_{x}(z)$ are set by (1.14) and (1.15), and $\Phi_{x}, \Psi_{x}$ are the solutions of integral equations (1.9) and (1.10), then

$$
\begin{gathered}
\int_{0}^{l}(1,-1) \Psi_{t} d F_{t} L_{t}^{*}(\bar{z})=-1-\frac{1}{2} R_{1} J\left(\frac{\overline{E_{0}(\bar{z})}}{\widetilde{E}_{0}(\bar{z})}\right), \\
\int_{0}^{l}(1,-1) \Psi_{t} d F_{t} \widetilde{L}_{t}^{*}(\bar{z})=\frac{1}{2} R_{1}\left(\begin{array}{c}
1 \\
1
\end{array}\right)+\frac{\overline{G_{l}(\bar{z})}-\overline{\widetilde{G}_{l}(\bar{z})}}{2}, \\
\int_{0}^{l}(1,1) \Phi_{t} d F_{t} L_{t}^{*}(\bar{z})=\frac{1}{2 z} R_{2}\left(\begin{array}{c}
1 \\
1
\end{array}\right)+\frac{\overline{E_{0}(\bar{z})}-\overline{\widetilde{E}_{0}(\bar{z})}}{2 z}, \\
\int_{0}^{l}(1,1) \Phi_{x} d F_{t} \widetilde{L}_{t}^{*}(\bar{z})=\frac{1}{2 z} R_{2} J\left(\frac{\overline{G_{l}(\bar{z})}}{\widetilde{G}_{l}(\bar{z})}\right)
\end{gathered}
$$


where $R_{1}$ and $R_{2}$ have the forms

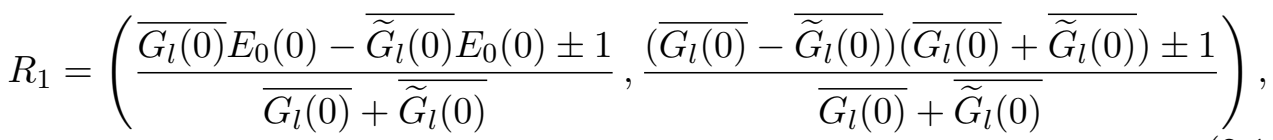

$$
\begin{aligned}
& R_{2}=\left(\frac{G_{l}(\infty) \overline{E_{0}(\bar{\infty})}-G_{l}^{2}(\infty)-G_{l}(\infty) \widetilde{G}_{l}(\infty) \pm 1}{\overline{G_{l}(\infty)}+\overline{\widetilde{G}_{l}(\infty)}},\right. \\
& \left.\frac{-3 G_{l}(\infty) \overline{E_{0}(\infty)}+\widetilde{G}_{l}^{2}(\infty)+G_{l}(\infty) \widetilde{G}_{l}(\infty)-2 \widetilde{G}_{l}(\infty) \overline{E_{0}(\infty)} \pm 1}{\overline{G_{l}(\infty)}+\widetilde{G}_{l}(\infty)}\right) .
\end{aligned}
$$

$\mathrm{P}$ r o o f. Let us consider the equation for the vector function $L_{x}(z)$

$$
(1-z) L_{x}(z)+2 z \int_{x}^{l} L_{t}(z) d F_{t} \Phi_{t}^{*} \Phi_{x}^{*-1} J=(1,1) \Psi_{x}
$$

and differentiate it by $x$ to get

$$
(1-z) L_{x}^{\prime}(z)-2 z L_{x}(z) a_{x} \Phi_{x}^{*} \Phi_{x}^{*-1} J+2 z \int_{x}^{l} L_{t}(z) d F_{t} \Phi_{t}^{*} \Phi_{x}^{*-1}\left(\Phi_{x}^{*-1}\right)^{\prime} J=(1,1) \Psi_{x}^{\prime} .
$$

Since $\Psi_{x}^{\prime}=\Psi_{x} a_{x} J$ and $\Phi_{x}^{\prime}=-\Phi_{x} a_{x} J$, then $\Phi_{x}^{* \prime}=-J a_{x} \Phi_{x}^{*}$ and $\left(\Phi_{x}^{*-1}\right)^{\prime}=$ $\Phi_{x}^{*-1} J a_{x}$. By using these statements, we obtain

$$
\begin{gathered}
(1-z) L_{x}^{\prime}(z)-2 z L_{x}(z) a_{x} J+\left((1,1) \Psi_{x}-(1-z) L_{x}(x)\right) a_{x} J=(1,1) \Psi_{x} a_{x} J \\
(1-z) L_{x}^{\prime}(z)=(1+z) L_{x}(z) a_{x} J
\end{gathered}
$$

i.e., $L_{x}^{\prime}(z)=\frac{1+z}{1-z} L_{x}(z) a_{x} J$ and $L_{x}^{* \prime}(z)=\frac{1+z}{1-z} J a_{x} L_{x}^{*}(z)$. Let us consider the following statements:

$$
\begin{gathered}
\left(\Psi_{x} J L_{x}^{*}(z)\right)^{\prime}=\Psi_{x} a_{x} J J L_{x}(z)+\Psi_{x} J \frac{1+\bar{z}}{1-\bar{z}} J a_{x} L_{x}(z)=\frac{2}{1-\bar{z}} \Psi_{x} a_{x} L_{x}^{*}(z), \\
\left((1,-1) \Psi_{x} J L_{x}^{*}(z)\right)^{\prime}=(1,-1) \frac{2}{1-\bar{z}} \Psi_{x} a_{x} L_{x}^{*}(z) .
\end{gathered}
$$

Since $\Psi_{l}=J, \Phi_{0}=I$, and $L_{l}(z)=(1,1) J \frac{1}{1-z}, L_{l}^{*}(\bar{z})=J\left(\begin{array}{c}1 \\ 1\end{array}\right) \frac{1}{1-z}, L_{0}(z)=$ $\frac{1}{1-z}\left(E_{0}(z), \widetilde{E}_{0}(z)\right)$ and $L_{0}^{*}(\bar{z})=\frac{1}{1-z}\left(\overline{E_{0}(\bar{z})}, \widetilde{E}_{0}(\bar{z})\right)$, then after integrating the statement

$$
(1,-1) \Psi_{x} a_{x} L_{x}^{*}(\bar{z})=\frac{1-z}{2}\left((1,-1) \Psi_{x} J L_{x}^{*}(\bar{z})\right),
$$


we obtain

$$
\begin{aligned}
\int_{0}^{l}(1,-1) \Psi_{x} d F_{x} L_{x}^{*}(\bar{z}) & =\frac{1-z}{2}(1,-1)\left(J J J\left(\begin{array}{c}
1 \\
1
\end{array}\right) \frac{1}{1-z}-\Psi_{0} J \frac{1}{1-z}\left(\frac{\overline{E_{0}(\bar{z})}}{\widetilde{E}_{0}(\bar{z})}\right)\right) \\
& =-1-\frac{1}{2}(1,-1) \Psi_{0} J\left(\frac{\overline{E_{0}(\bar{z})}}{\widetilde{E}_{0}(\bar{z})}\right) .
\end{aligned}
$$

Similarly, using $\left(\Phi_{x} J L_{x}^{*}(\bar{z})\right)^{\prime}=\frac{2 z}{1-z} \Phi_{x} a_{x} L_{x}^{*}(\bar{z})$, we integrate the following statement:

$$
\begin{aligned}
& \int_{0}^{l}(1,1) \Phi_{t} d F_{t} L_{t}^{*}(\bar{z})=\frac{1-z}{2 z}(1,1)\left(\Phi_{l} J J\left(\begin{array}{c}
1 \\
1
\end{array}\right) \frac{1}{1-z}-I J \frac{1}{1-z}\left(\overline{E_{0}(\bar{z})}, \overline{\left.\widetilde{E}_{0}(\bar{z})\right)}\right)\right. \\
& =\frac{1}{2 z}(1,1)\left(\Phi_{l}\left(\begin{array}{c}
1 \\
1
\end{array}\right)-J\left(\overline{E_{0}(\bar{z})}, \overline{\widetilde{E}_{0}(\bar{z})}\right)\right)=\frac{1}{2 z}(1,1) \Phi_{l}\left(\begin{array}{c}
1 \\
1
\end{array}\right)+\frac{\overline{E_{0}(\bar{z})}-\overline{\widetilde{E}_{0}(\bar{z})}}{2 z} .
\end{aligned}
$$

Now we take the equation for the vector function $\widetilde{L}_{x}(z)$,

$$
(1-z) \widetilde{L}_{x}(z)+2 z \int_{0}^{l} \widetilde{L}_{t}(z) d F_{t} J \Phi_{t}^{-1} \Phi_{x}=(1,-1) \Phi_{x}
$$

and differentiate it by $x$ to get

$$
\begin{gathered}
(1-z) \widetilde{L}_{x}^{\prime}(z)+2 z \widetilde{L}_{x}(z) a_{x} J \Phi_{x}^{-1} \Phi_{x}-2 z \int_{0}^{l} \widetilde{L}_{t}(z) d F_{t} J \Phi_{t}^{-1} \Phi_{x} a_{x} J=-(1,-1) \Phi_{x} a_{x} J, \\
(1-z) \widetilde{L}_{x}^{\prime}(z)+2 z \widetilde{L}_{x}(z) a_{x} J+\left((1-z) \widetilde{L}_{x}(z)-(1,-1) \Phi_{x}\right) a_{x} J=-(1,-1) \Phi_{x} a_{x} J \\
(1-z) \widetilde{L}_{x}^{\prime}(z)+(1+z) \widetilde{L}_{x}(z) a_{x} J=0 .
\end{gathered}
$$

Thus, $\widetilde{L}_{x}^{\prime}(z)=-\frac{1+z}{1-z} \widetilde{L}_{x}(z) a_{x} J$ and $\left(\widetilde{L}_{x}^{*}(z)\right)^{\prime}=-\frac{1+z}{1-z} J a_{x} \widetilde{L}_{x}^{*}(z)$. Let us consider the following statements:

$$
\left(\Psi_{x} J \widetilde{L}_{x}^{*}(\bar{z})\right)^{\prime}=\Psi_{x} a_{x} J J \widetilde{L}_{x}^{*}(\bar{z})-\Psi_{x} J \frac{1+z}{1-z} J a_{x} \widetilde{L}_{x}^{*}(\bar{z})=\frac{-2 z}{1-z} \Psi_{x} a_{x} \widetilde{L}_{x}^{*}(\bar{z}) .
$$

And after integration we obtain

$$
\int_{0}^{l}(1,-1) \Psi_{x} a_{x} \widetilde{L}_{x}^{*}(\bar{z})=\frac{1-z}{-2 z}(1,-1)\left(J J \frac{1}{1-z}\left(\frac{\overline{G_{l}(\bar{z})}}{\widetilde{G}_{l}(\bar{z})}\right)-\Psi_{x} J \frac{1}{1-z} I\left(\begin{array}{c}
1 \\
-1
\end{array}\right)\right)
$$




$$
=\frac{1}{-2 z}(1,-1)\left(\frac{\overline{G_{l}(\bar{z})}}{\widetilde{G}_{l}(\bar{z})}\right)-\frac{1}{-2 z}(1,-1) \Psi_{0}\left(\begin{array}{c}
-1 \\
-1
\end{array}\right) .
$$

Hence,

$$
\int_{0}^{l}(1,-1) \Psi_{x} a_{x} \widetilde{L}_{x}^{*}(\bar{z})=-\frac{1}{2 z}(1,-1) \Psi_{0}\left(\begin{array}{c}
1 \\
1
\end{array}\right)-\frac{\overline{G_{l}(\bar{z})}-\overline{\widetilde{G}_{l}(\bar{z})}}{2 z} .
$$

Similarly, we can get

$$
\left(\Phi_{x} J \widetilde{L}_{x}^{*}(\bar{z})\right)^{\prime}=\frac{2 z}{1-z} \Phi_{x} a_{x} \widetilde{L}_{x}^{*}(\bar{z})
$$

then

$$
\int_{0}^{l}(1,1) \Phi_{x} a_{x} \widetilde{L}_{x}^{*}(\bar{z})=\frac{1-z}{2 z}(1,1)\left(\Phi_{l} J \frac{1}{1-z}\left(\frac{\overline{G_{l}(\bar{z})}}{\widetilde{G}_{l}(\bar{z})}\right)-I J \frac{1}{1-z} I\left(\begin{array}{c}
1 \\
-1
\end{array}\right)\right),
$$

and

$$
\int_{0}^{l}(1,1) \Phi_{x} a_{x} \widetilde{L}_{x}^{*}(\bar{z})=\frac{1}{2 z} \Phi_{l} J\left(\frac{\overline{G_{l}(\bar{z})}}{\widetilde{G}_{l}(\bar{z})}\right)+\frac{1}{z} .
$$

Write down a characteristic matrix-function $S_{\triangle}(z)$ element-wisely, $S_{\triangle}(z)=\left(\begin{array}{cc}a(z) & b(z) \\ c(z) & d(z)\end{array}\right)$, and find its coefficients. Since $N_{0}(z)=-S_{\triangle}(z)$, $\widetilde{N}_{l}^{*}(\bar{z})=S_{\triangle}(z),(1,1) N_{x}(z) J=\left(E_{0}(z), \widetilde{E}_{0}(z)\right)$ and $(1,-1) \widetilde{N}_{l}(z)=\left(G_{l}(z), \widetilde{G}_{l}(z)\right)$, then $\widetilde{N}_{l}^{*}(z)\left(\begin{array}{c}1 \\ -1\end{array}\right)=\left(\frac{\overline{G_{l}(\bar{z})}}{\widetilde{G}_{l}(\bar{z})}\right)$.

For $S_{\triangle}(z)$, we get the equations

$$
\begin{gathered}
-(1,1)\left(\begin{array}{cc}
a(z) & b(z) \\
c(z) & d(z)
\end{array}\right) J=\left(E_{0}(z), \widetilde{E}_{0}(z)\right), \\
\left(\begin{array}{cc}
a(z) & b(z) \\
c(z) & d(z)
\end{array}\right)\left(\begin{array}{c}
1 \\
-1
\end{array}\right)=\left(\begin{array}{c}
\overline{G_{l}(\bar{z})} \\
\widetilde{G}_{l}(\bar{z})
\end{array}\right) .
\end{gathered}
$$

By solving this system, we obtain the coefficients of the matrix-function $S_{\triangle}(z)$ :

$$
c(z)=E_{0}(z)-a(z), \quad b(z)=a(z)-\overline{G_{l}(\bar{z}),} \quad d(z)=E_{0}(z)-\overline{\widetilde{G}_{l}(\bar{z})}-a(z) .
$$


Now we will use the condition $\left|\operatorname{det} S_{\triangle}(z)\right|^{2}=1$, i.e., $\left|\operatorname{det} S_{\triangle}(z)\right|= \pm 1$, or $a(z) d(z)-b(z) c(z)= \pm 1$, to get the expression for $a(z)$

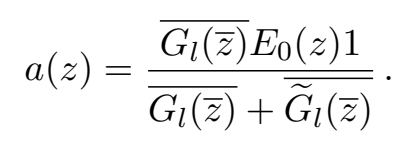

Now we can find the expression of $(1,-1) \Psi_{x}$ :

$$
\begin{gathered}
(1,-1) \Psi_{x}=N_{0}(0) J=-(1,-1) S_{\triangle}(0) J \\
=\left(\frac{\overline{G_{l}(0)} E_{0}(0)-\overline{\widetilde{G}_{l}(0)} E_{0}(0) \pm 1}{\overline{G_{l}(0)}+\overline{\widetilde{G}_{l}(0)}}, \frac{\left(\overline{G_{l}(0)}-\overline{\widetilde{G}_{l}(0)}\right)\left(\overline{G_{l}(0)}+\overline{\widetilde{G}_{l}(0)}\right) \pm 1}{\overline{G_{l}(0)}+\overline{\widetilde{G}_{l}(0)}}\right)
\end{gathered}
$$

and the expression of $(1,1) \Phi_{l}$ :

$$
\begin{gathered}
(1,1) \Phi_{l}=(1,1) \widetilde{N}_{l}(\infty)=\left(\frac{G_{l}(\infty) \overline{E_{0}(\bar{\infty})}-G_{l}^{2}(\infty)-G_{l}(\infty) \widetilde{G}_{l}(\infty) \pm 1}{\overline{G_{l}(\infty)}+\overline{\widetilde{G}_{l}(\infty)}},\right. \\
\left.\frac{-3 G_{l}(\infty) \overline{E_{0}(\infty)}+\widetilde{G}_{l}^{2}(\infty)+G_{l}(\infty) \widetilde{G}_{l}(\infty)-2 \widetilde{G}_{l}(\infty) \overline{E_{0}(\infty)} \pm 1}{\overline{G_{l}(\infty)}+\overline{\widetilde{G}_{l}(\infty)}}\right) .
\end{gathered}
$$

Having defined these expressions as $R_{1}$ and $R_{2}$, respectively, and using integrals (2.12)-(2.15), we obtain the expressions stated in the lemma definition.

Lemma 6. The operator $T_{1}^{*}$ affects the vector function $L_{x}(z)(1.14)$ in the following way:

$$
T_{1}^{*} L_{x}(z)=(z+\mu(z)) L_{x}(z)+\nu(z) \widetilde{L}_{x}(z)+\frac{E_{0}(z)-\widetilde{E}_{0}(z)}{2} \widetilde{L}_{x}(0),
$$

where

$$
\begin{aligned}
& \nu(z)=\frac{c_{2}(z) c_{3}(z)-c_{1}(z) c_{4}(z)}{c_{2}(z)-c_{4}(z)}, \\
& \mu(z)=\frac{c_{1}(z)-c_{3}(z)}{c_{2}(z)-c_{4}(z)}, \\
& c_{1}(z)=\frac{\left(E_{0}(z)+\widetilde{E}_{0}(z)\right)\left(1-z^{2}\right)}{2\left(E_{0}(z) \overline{E_{0}(\bar{z})}-\widetilde{E}_{0}(z) \overline{\widetilde{E}_{0}(\bar{z})}\right)}\left(\frac{1}{2 z} R_{2}\left(\begin{array}{c}
1 \\
1
\end{array}\right)+\frac{\overline{E_{0}(\bar{z})}-\overline{\widetilde{E}_{0}(\bar{z})}}{2 z}\right), \\
& c_{2}(z)=\frac{\left(G_{l}^{\prime}(z)+\widetilde{G}_{l}^{\prime}(z)\right)\left(1-z^{2}\right)}{2\left(E_{0}(z) \overline{E_{0}(\bar{z})}-\widetilde{E}_{0}(z) \overline{\widetilde{E}_{0}(\bar{z})}\right)}, \\
& c_{3}(z)=\frac{E_{0}(z)+\widetilde{E}_{0}(z)}{E_{0}^{\prime}(z)-\widetilde{E}_{0}^{\prime}(z)}\left(\frac{1}{2 z} R_{2} J\left(\frac{\overline{G_{l}(\bar{z})}}{\widetilde{G}_{l}(\bar{z})}\right)\right),
\end{aligned}
$$




$$
c_{4}(z)=\frac{2\left(G_{l}(z) \overline{G_{l}(\bar{z})}-\widetilde{G}_{l}(z) \overline{\widetilde{G}_{l}(\bar{z})}\right)}{\left(E_{0}^{\prime}(z)-\widetilde{E}_{0}^{\prime}(z)\right)\left(1-z^{2}\right)},
$$

and $R_{1}, R_{2}$ have the forms of (2.10) and (2.11), respectively.

P r o o f. According to Lemma 4,

$$
T_{1}^{*} L_{x}(z)=z L_{x}(z)+\frac{E_{0}(z)-\widetilde{E}_{0}(z)}{2} \widetilde{L}_{x}(0)+\frac{E_{0}(z)+\widetilde{E}_{0}(z)}{2}(1,1) \Phi_{x} .
$$

Now we will show that the vectors $L_{x}(z)$ and $\widetilde{L}_{x}(z)$ are linearly independent with each fixed $x \in[0, l]$ and any $z \in C$. Assuming the opposite, $\delta(z) L_{x}(z)=\widetilde{L}_{x}(z)$, let us suppose that

$$
\delta(z)(1-z T)^{-1} \Phi(1,1)=\left(1-z T^{*}\right)^{-1} \Psi^{*}(1,-1) .
$$

Apply the operator $T_{1}$ to both parts of the equation

$$
\begin{gathered}
\delta(z) \frac{(1-z T)^{-1} \Phi(1,1)-\Phi(1,1)}{z}=z\left(1-z T^{*}\right)^{-1} \Psi^{*}(1,-1)-\Phi J S^{*}\left(\frac{1}{\bar{z}}\right)(1,-1), \\
(1-z T)^{-1} \Phi(1,1)\left(\delta(z)-z^{2}\right)=\Phi(1,1) \delta(z)+z \Phi J S^{*}\left(\frac{1}{\bar{z}}\right)(1,-1) .
\end{gathered}
$$

Let us consider the case where $\delta(z)=z^{2}$. From the previous equation we obtain that $(1,-1) S^{*}\left(\frac{1}{\bar{z}}\right)=-z(1,-1)$, which is impossible because $S^{*}\left(\frac{1}{\bar{z}}\right)=K^{*}+z \Psi^{*}(1-$ $\left.z T_{1}^{*}\right)^{-1} \Phi^{*}$ and $S^{*}\left(\frac{1}{\bar{z}}\right) \neq 0$ where $z=0$.

If $\delta(z) \neq z^{2}$, then

$$
(1-z T)^{-1} \Phi(1,1)=\Phi\left(\frac{(1,1) \delta(z)+z J S^{*}\left(\frac{1}{\bar{z}}\right)(1,-1)}{\delta(z)-z^{2}}\right)
$$

and $(1-z T)^{-1} \Phi(1,1) \in \Phi E$ for $\forall z$, but $L_{x}(z) \notin \Phi E$ for $\forall z$.

Thus the functions $L_{x}(z)$ and $\widetilde{L}_{x}(z)$ are linearly independent and form basis in $E^{2}$ for each fixed $x$ for $\forall z$. Therefore we present the last term in the form

$$
\frac{E_{0}(z)+\widetilde{E}_{0}(z)}{2}(1,1) \Phi_{x}=\mu(z) L_{x}(z)+\nu(z) \widetilde{L}_{x}(z)
$$

subsequently multiplying $(2.23)$ by $\widetilde{L}_{x}^{*}(z)$,

$$
\frac{E_{0}(z)+\widetilde{E}_{0}(z)}{2} \int_{0}^{l}(1,1) \Phi_{x} d F_{t} \widetilde{L}_{t}^{*}(\bar{z})=\mu(z) \int_{0}^{l} L_{t}(z) d F_{t} \widetilde{L}_{t}^{*}(\bar{z})+\nu(z) \int_{0}^{l} \widetilde{L}_{t}(z) d F_{t} \widetilde{L}_{t}^{*}(\bar{z}) .
$$


Let us calculate the integrals in the above statement. First we get

$$
\begin{aligned}
& N_{0}(z)-\widetilde{N}_{l}^{*}(\omega)=2(\bar{\omega}-z) \int_{0}^{l} M_{t}(z) d F_{t} \widetilde{M}_{t}^{*}(\omega), \\
& \widetilde{N}_{l}(z)-N_{0}^{*}(\omega)=2(z-\bar{\omega}) \int_{0}^{l} \widetilde{M}_{t}(z) d F_{t} M_{t}^{*}(\omega) .
\end{aligned}
$$

We multiply $(2.25)$ on the left by $(-1,1)$ and on the right by $(1,1)^{T}$. Since $(-1,1) \widetilde{N}_{l}(z)=\left(G_{l}(z), \widetilde{G}_{l}(z)\right)$ and $(1,1) N_{0}(\omega)=\left(E_{0}(\omega), \widetilde{E}_{0}(\omega)\right)$, then

$$
G_{l}(z)+\widetilde{G}_{l}(z)+\overline{E_{0}(\omega)}-\overline{\widetilde{E}_{0}(\omega)}=2(z-\bar{\omega}) \int_{0}^{l} \widetilde{L}_{t}(z) d F_{t} L_{t}^{*}(\omega) .
$$

Write the expression in the form

$$
(z-\omega) \int_{0}^{l} \widetilde{L}_{t}(z) d F_{t} L_{t}^{*}(\bar{\omega})=\frac{G_{l}(z)+\widetilde{G}_{l}(z)}{2}+\frac{\overline{\widetilde{E}_{0}(\bar{\omega})}-\overline{E_{0}(\bar{\omega})}}{2} .
$$

Let us define $f(z)=\frac{G_{l}(z)+\widetilde{G}_{l}(z)}{2}$ and $g(\omega)=\frac{\overline{\widetilde{E}_{0}(\bar{\omega})}-\overline{E_{0}(\bar{\omega})}}{2}$. Since $f(z)=-g(z)$, then

$$
\begin{gathered}
\frac{f(z)-g(\omega)}{z-\omega} \rightarrow f^{\prime}(z), \quad \omega \mapsto z, \\
\int_{0}^{l} \widetilde{L}_{t}(z) d F_{t} L_{t}^{*}(\bar{z})=\frac{1}{2}\left(\frac{d G_{l}(z)}{d z}+\frac{d \widetilde{G}_{l}(z)}{d z}\right) .
\end{gathered}
$$

Now, if we multiply $(2.24)$ on the left by $(1,1)$ and on the right by $(-1,1)^{T}$, then

$$
\begin{gathered}
\left(E_{0}(z), \widetilde{E}_{0}(z)\right)\left(\begin{array}{c}
-1 \\
1
\end{array}\right)-(1,1)\left(\frac{\overline{G_{l}(\omega)}}{\widetilde{G}_{l}(\omega)}\right)=2(\bar{\omega}-z) \int_{0}^{l} L_{t}(z) d F_{t} \widetilde{L}_{t}^{*}(\omega), \\
E_{0}(z)-\widetilde{E}_{0}(z)+\overline{G_{l}(\omega)}+\overline{\widetilde{G}_{l}(\omega)}=2(z-\bar{\omega}) \int_{0}^{l} L_{t}(z) d F_{t} \widetilde{L}_{t}^{*}(\omega)
\end{gathered}
$$

and, similarly,

$$
\int_{0}^{l} L_{t}(z) d F_{t} \widetilde{L}_{t}^{*}(\bar{z})=\frac{1}{2}\left(\frac{d E_{l}(z)}{d z}-\frac{d \widetilde{E}_{l}(z)}{d z}\right)
$$


We also have the expressions for two integrals:

$$
\begin{aligned}
& \int_{0}^{l} \widetilde{L}_{t}(z) d F_{t} \widetilde{L}_{t}^{*}(\bar{\omega})=\frac{G_{x}(z) \overline{G_{x}(\bar{\omega})}-\widetilde{G}_{x}(z) \overline{\widetilde{G}_{x}(\bar{\omega})}}{1-z \bar{\omega}}, \\
& \int_{0}^{l} L_{t}(z) d F_{t} L_{t}^{*}(\bar{\omega})=\frac{E_{0}(z) \overline{E_{0}(\bar{\omega})}-\widetilde{E}_{0}(z) \overline{\widetilde{E}_{0}(\bar{\omega})}}{1-z \bar{\omega}} .
\end{aligned}
$$

By using (2.26)-(2.29), we obtain

$$
\begin{gathered}
\frac{E_{0}(z)+\widetilde{E}_{0}(z)}{2} \int_{0}^{l}(1,1) \Phi_{x} d F_{t} \widetilde{L}_{t}^{*}(\bar{z}) \\
=\nu(z)\left(\frac{E_{l}^{\prime}(z)-\widetilde{E}_{l}^{\prime}(z)}{2}\right)+\mu(z)\left(\frac{G_{x}(z) \overline{G_{x}(\bar{z})}-\widetilde{G}_{x}(z) \overline{G_{x}(\bar{z})}}{1-z^{2}}\right) .
\end{gathered}
$$

Now we multiply statement $(2.23)$ on the right by $L_{x}^{*}(\bar{z})$,

$$
\frac{E_{0}(z)+\widetilde{E}_{0}(z)}{2} \int_{0}^{l}(1,1) \Phi_{x} d F_{t} L_{t}^{*}(\bar{z})=\nu(z) \int_{0}^{l} L_{t}(z) d F_{t} L_{t}^{*}(\bar{z})+\mu(z) \int_{0}^{l} \widetilde{L}_{t}(z) d F_{t} L_{t}^{*}(\bar{z}) .
$$

By using expressions (2.26)-(2.29), in a similar way, we obtain

$$
\begin{aligned}
& \frac{E_{0}(z)+\widetilde{E}_{0}(z)}{2} \int_{0}^{l}(1,1) \Phi_{x} d F_{t} L_{t}^{*}(\bar{z}) \\
& =\nu(z)\left(\frac{E_{0}(z) \overline{E_{0}(\bar{z})}-\widetilde{E}_{0}(z) \overline{\widetilde{E}_{0}(\bar{z})}}{1-z^{2}}\right)+\mu(z)\left(\frac{G_{l}^{\prime}(z)+\widetilde{G}_{l}^{\prime}(z)}{2}\right) .
\end{aligned}
$$

Now let us calculate $\nu(z)$ and $\mu(z)$. Taking into account (2.10) and (2.11), we will define the coefficients:

$$
\begin{gathered}
c_{1}(z)=\frac{\left(E_{0}(z)+\widetilde{E}_{0}(z)\right)\left(1-z^{2}\right)}{2\left(E_{0}(z) \overline{E_{0}(\bar{z})}-\widetilde{E}_{0}(z) \overline{\widetilde{E}_{0}(\bar{z})}\right)}\left(\frac{1}{2 z} R_{2}\left(\begin{array}{c}
1 \\
1
\end{array}\right)+\frac{\overline{E_{0}(\bar{z})}-\overline{\widetilde{E}_{0}(\bar{z})}}{2 z}\right), \\
c_{2}(z)=\frac{\left(G_{l}^{\prime}(z)+\widetilde{G}_{l}^{\prime}(z)\right)\left(1-z^{2}\right)}{2\left(E_{0}(z) \overline{E_{0}(\bar{z})}-\widetilde{E}_{0}(z) \overline{\widetilde{E}_{0}(\bar{z})}\right.}, \\
c_{3}(z)=\frac{E_{0}(z)+\widetilde{E}_{0}(z)}{E_{0}^{\prime}(z)-\widetilde{E}_{0}^{\prime}(z)}\left(\frac{1}{2 z} R_{2} J\left(\frac{\overline{G_{l}(\bar{z})}}{\widetilde{G}_{l}(\bar{z})}\right)\right),
\end{gathered}
$$




$$
c_{4}(z)=\frac{2\left(G_{l}(z) \overline{G_{l}(\bar{z})}-\widetilde{G}_{l}(z) \overline{\widetilde{G}_{l}(\bar{z})}\right)}{\left(E_{0}^{\prime}(z)-\widetilde{E}_{0}^{\prime}(z)\right)\left(1-z^{2}\right)} .
$$

Hence,

$$
\nu(z)=\frac{c_{2}(z) c_{3}(z)-c_{1}(z) c_{4}(z)}{c_{2}(z)-c_{4}(z)}, \quad \mu(z)=\frac{c_{1}(z)-c_{3}(z)}{c_{2}(z)-c_{4}(z)}
$$

and thus the expression $c_{2}(z)-c_{4}(z)$ is not identically equal to zero. Finally we get

$$
T^{*} L_{x}(z)=z L_{x}(z)+\frac{E_{0}(z)-\widetilde{E}_{0}(z)}{2} \widetilde{L}_{x}(0)+\mu(z) L_{x}(z)+\nu(z) \widetilde{L}_{x}(z),
$$

which proves the lemma.

Lemma 7. The operator $T_{1}$ affects the vector function $\widetilde{L}_{x}(z)(1.15)$ in the following way:

$$
T_{1} \widetilde{L}_{x}(z)=(z-\widetilde{\nu}(z)) \widetilde{L}_{x}(z)+\frac{\widetilde{G}_{l}(z)-G_{l}(z)}{2} L_{x}(0)-\widetilde{\mu}(z) L_{x}(z),
$$

where

$$
\begin{gathered}
\widetilde{\nu}(z)=\frac{c_{2}(z) \widetilde{c}_{3}(z)-\widetilde{c}_{1}(z) c_{4}(z)}{c_{2}(z)-c_{4}(z)} \\
\widetilde{\mu}(z)=\frac{\widetilde{c}_{1}(z)-\widetilde{c}_{3}(z)}{c_{2}(z)-c_{4}(z)} \\
\widetilde{c}_{1}(z)=\frac{\left(E_{0}(z)+\widetilde{E}_{0}(z)\right)\left(1-z^{2}\right)}{2\left(E_{0}(z) \overline{E_{0}(\bar{z})}-\widetilde{E}_{0}(z) \overline{\widetilde{E}_{0}(\bar{z})}\right)}\left(-1-\frac{1}{2} R_{1} J\left(\frac{\overline{E_{0}(\bar{z})}}{\widetilde{E}_{0}(\bar{z})}\right)\right), \\
\widetilde{c}_{3}(z)=\frac{E_{0}(z)+\widetilde{E}_{0}(z)}{E_{0}^{\prime}(z)-\widetilde{E}_{0}^{\prime}(z)}\left(\frac{1}{2} R_{1}\left(\begin{array}{c}
1 \\
1
\end{array}\right)+\frac{\left.\overline{G_{l}(\bar{z})}-{\widetilde{G_{l}}(\bar{z})}_{2}\right)}{2}\right.
\end{gathered}
$$

and $c_{2}(z), c_{4}(z)$ are (2.20) and (2.22), and $R_{1}, R_{2}$ are (2.10) and (2.11), respectively.

P r o o f. According to Lemma 2,

$$
T_{1} \widetilde{L}_{x}(z)=z \widetilde{L}_{x}(z)+\frac{\widetilde{G}_{l}(z)-G_{l}(z)}{2} L_{x}(0)-\frac{\widetilde{G}_{l}(z)+G_{l}(z)}{2}(1,-1) \Psi_{x}
$$

We can perform the calculations that are similar to those made in Lemma 5 . Since the functions $L_{x}(z)$ and $\widetilde{L}_{x}(z)$ are linearly independent and form the basis in $L_{2}$, we can present the latter term of the above statement in the form

$$
\frac{\widetilde{G}_{l}(z)+G_{l}(z)}{2}(1,-1) \Psi_{x}=\widetilde{\mu}(z) L_{x}(z)+\widetilde{\nu}(z) \widetilde{L}_{x}(z) .
$$


Similarly, we multiply it by $L_{x}(z)$ and $\widetilde{L}_{x}(z)$ and using the expressions for $(2.26)-$ (2.29), we obtain

$$
\begin{gathered}
\frac{\widetilde{G}_{l}(z)+G_{l}(z)}{2} \int_{0}^{l}(1,-1) \Psi_{t} d F_{t} \widetilde{L}_{t}^{*}(\bar{z}) \\
=\widetilde{\nu}(z)\left(\frac{E_{l}^{\prime}(z)-\widetilde{E}_{l}^{\prime}(z)}{2}\right)+\widetilde{\mu}(z) l\left(\frac{G_{x}(z) \overline{G_{x}(\bar{z})}-\widetilde{G}_{x}(z) \widetilde{\widetilde{G}_{x}(\bar{z})}}{1-z^{2}}\right), \\
\frac{\widetilde{G}_{l}(z)+G_{l}(z)}{2} \int_{0}^{l}(1,-1) \Psi_{t} d F_{t} L_{t}^{*}(\bar{z}) \\
=\widetilde{\nu}(z)\left(\frac{E_{0}(z) \overline{E_{0}(\bar{z})}-\widetilde{E}_{0}(z) \overline{\widetilde{E}_{0}(\bar{z})}}{1-z^{2}}\right)+\widetilde{\mu}(z)\left(\frac{G_{l}^{\prime}(z)+\widetilde{G}_{l}^{\prime}(z)}{2}\right) .
\end{gathered}
$$

By using (2.10) and (2.11) and introducing similar coefficients, we obtain

$$
\begin{gathered}
\widetilde{c}_{1}(z)=\frac{\left(E_{0}(z)+\widetilde{E}_{0}(z)\right)\left(1-z^{2}\right)}{2\left(E_{0}(z) \overline{E_{0}(\bar{z})}-\widetilde{E}_{0}(z) \overline{\widetilde{E}_{0}(\bar{z})}\right)}\left(-1-\frac{1}{2} R_{1} J\left(\frac{\overline{E_{0}(\bar{z})}}{\widetilde{E}_{0}(\bar{z})}\right)\right), \\
\widetilde{c}_{2}(z)=\frac{\left(G_{l}^{\prime}(z)+\widetilde{G}_{l}^{\prime}(z)\right)\left(1-z^{2}\right)}{2\left(E_{0}(z) \overline{E_{0}(\bar{z})}-\widetilde{E}_{0}(z) \overline{\widetilde{E}_{0}(\bar{z})}\right)}=c_{2}(z), \\
\widetilde{c}_{3}(z)=\frac{E_{0}(z)+\widetilde{E}_{0}(z)}{E_{0}^{\prime}(z)-\widetilde{E}_{0}^{\prime}(z)}\left(\frac{1}{2} R_{1}\left(\begin{array}{c}
1 \\
1
\end{array}\right)+\frac{\overline{G_{l}(\bar{z})}-\overline{\widetilde{G}_{l}(\bar{z})}}{2}\right), \\
\widetilde{c}_{4}(z)=\frac{2\left(G_{l}(z) \overline{G_{l}(\bar{z})}-\widetilde{G}_{l}(z) \overline{\widetilde{G}_{l}(\bar{z})}\right)}{\left(E_{0}^{\prime}(z)-\widetilde{E}_{0}^{\prime}(z)\right)\left(1-z^{2}\right)}=c_{4}(z) .
\end{gathered}
$$

Thus, for $\widetilde{\nu}(z)$ and $\widetilde{\mu}(z)$ we get

$$
\begin{gathered}
\widetilde{\nu}(z)=\frac{c_{2}(z) \widetilde{c}_{3}(z)-\widetilde{c}_{1}(z) c_{4}(z)}{c_{2}(z)-c_{4}(z)}, \\
\widetilde{\mu}(z)=\frac{\widetilde{c}_{1}(z)-\widetilde{c}_{3}(z)}{c_{2}(z)-c_{4}(z)} .
\end{gathered}
$$

Finally we obtain the expression

$$
T_{1} \widetilde{L}_{x}(z)=z \widetilde{L}_{x}(z)+\frac{\widetilde{G}_{l}(z)-G_{l}(z)}{2} L_{x}(0)-\widetilde{\mu}(z) L_{x}(z)-\widetilde{\nu}(z) \widetilde{L}_{x}(z),
$$

which proves the lemma. 


\section{De Branges Transformation}

In [9], the following results were obtained, namely Lemmas 8-10.

Lemma 8. [9] De Branges transformation $B_{L}$ (Definition 1) affects $T_{1} f$ in the following way:

$$
B_{L}\left(T_{1} f\right)=(z+\overline{\mu(\bar{z})}) F_{1}(z)+\nu(\bar{z}) F_{2}(z)+\frac{\overline{E_{0}(\bar{z})}-\overline{\widetilde{E}_{0}(\bar{z})}}{2} F_{2}(0),
$$

where $F_{1}$ and $F_{2}$ have the same form as in (1.16).

Lemma 9. [9] De Branges transformation $B_{\widetilde{L}}$ affects $T_{1} f$ in the following way:

$$
B_{\widetilde{L}}\left(T_{1} f\right)=\frac{F_{2}(z)-F_{2}(0)}{z},
$$

where $F_{1}$ and $F_{2}$ have the same form as in (1.16).

Lemma 10. [9] If the vector $(1,-1)$ is latent for $\widetilde{N}^{*}+z \widetilde{\Gamma}^{*}$ with each $z$, then de Branges transformation $B_{\widetilde{L}}$ affects $T_{2} f$, where $T_{2} f$ is from the knot $\triangle(1.21)$, in the following way:

$$
B_{\widetilde{L}}\left(T_{2} f(z)\right)=\frac{F_{2}(z) n(z)-F(0) n(0)}{z},
$$

where $F_{1}$ and $F_{2}$ have the form of (1.16), and the function $n(z)$ satisfies the statement

$$
\left(\widetilde{N}^{*}+z \widetilde{\Gamma}^{*}\right)(1,-1)=n(z)(1,-1) .
$$

Let us prove the lemma below.

Lemma 11. If the vector $(1,1)$ is latent for $(N+z \Gamma)$, then de Branges transformation $B_{L}$ affects $T_{2} f$, where $T_{2} f$ is from the knot $\triangle(1.21)$, in the following way:

$$
B_{L}\left(T_{2} f(z)\right)=\frac{F_{1}(z)}{m(z)}+\frac{\widetilde{\mu}(z)}{m(z)} F_{1}(z)+\frac{\widetilde{\nu}(z)}{m(z)} F_{2}(z),
$$

where $F_{1}$ and $F_{2}$ have the form of (1.16), and the function $m(z)$ satisfies the statement

$$
(N+z \Gamma)^{-1}(1,1)=\frac{1}{m(z)}(1,1) .
$$

Therefore the coefficients $\widetilde{\mu}(z)$ and $\widetilde{\nu}(z)$ have the forms

$$
\widetilde{\mu}(z)=\frac{I_{1}(z) d_{3}(z)-I_{2}(z) d_{1}(z)}{d_{2}(z) d_{3}(z)-d_{1}(z) d_{4}(z)}
$$




$$
\widetilde{\nu}(z)=\frac{I_{1}(z) d_{4}(z)-I_{2}(z) d_{2}(z)}{d_{1}(z) d_{4}(z)-d_{2}(z) d_{3}(z)},
$$

where

$$
\begin{gathered}
I_{1}(z)=\frac{1}{2 z}(1,1) \sqrt{2} S\left(\frac{1}{z}\right) \widetilde{\sigma}_{2}\left(\Phi_{l}\left(\begin{array}{c}
1 \\
1
\end{array}\right)-J\left(\overline{E_{0}(\bar{z})}, \overline{\widetilde{E}_{0}(\bar{z})}\right)\right), \\
I_{2}(z)=\frac{1}{2 z}(1,1) \sqrt{2} S\left(\frac{1}{z}\right) \widetilde{\sigma}_{2}\left(\Phi_{l} J\left(\frac{\overline{G_{l}(\bar{z})}}{\widetilde{G}_{l}(\bar{z})}\right)+\left(\begin{array}{c}
1 \\
1
\end{array}\right)\right), \\
d_{1}(z)=\frac{E_{0}(z) \overline{E_{0}(\bar{z})}-\widetilde{E}_{0}(z) \overline{\widetilde{E}_{0}(\bar{z})}}{1-|z|^{2}} \\
d_{2}(z)=\frac{G_{l}^{\prime}(z)+\widetilde{G}_{l}^{\prime}(z)}{2} \\
d_{3}(z)=\frac{E_{0}^{\prime}(z)-\widetilde{E}_{0}^{\prime}(z)}{2} \\
d_{4}(z)=\frac{G_{l}(z) \overline{G_{l}(\bar{z})}-\widetilde{G}_{l}(z) \overline{\widetilde{G}_{l}(\bar{z})}}{1-|z|^{2}} .
\end{gathered}
$$

P r o o f. Using the expressions for $T_{1}(1.23)$ and $T_{2}$ (1.24), we obtain

$$
\begin{gathered}
T_{2} \Phi=T_{1} \Phi N+\Phi \Gamma, \\
z T_{2} \Phi=z T_{1} \Phi N+z \Phi \Gamma=\left(z T_{1}-1\right) \Phi N+\Phi(\Gamma z+N), \\
z\left(z T_{1}-1\right)^{-1} T_{2} \Phi=\Phi N+\left(z T_{1}-1\right)^{-1} \Phi(\Gamma z+N), \\
T_{2}^{*} T_{2} z\left(z T_{1}-1\right)^{-1} \Phi=T_{2}^{*} \Phi N+T_{2}^{*}\left(z T_{1}-1\right)^{-1} \Phi(\Gamma z+N) .
\end{gathered}
$$

Due to the knots relations, we get the statement $T_{2}^{*} T_{2}+\Psi^{*} \widetilde{\sigma}_{2} \Psi=I$. Then

$$
\begin{gathered}
\left(I-\Psi^{*} \widetilde{\sigma}_{2} \Psi\right) z\left(z T_{1}-1\right)^{-1} \Phi=T_{2}^{*} \Phi N+T_{2}^{*}\left(z T_{1}-1\right)^{-1} \Phi(\Gamma z+N), \\
\left(\Psi^{*} \widetilde{\sigma}_{2} \Psi-I\right) z\left(1-z T_{1}\right)^{-1} \Phi=T_{2}^{*} \Phi N+T_{2}^{*}\left(z T_{1}-1\right)^{-1} \Phi(\Gamma z+N), \\
z \Psi^{*} \widetilde{\sigma}_{2} \Psi z\left(1-z T_{1}\right)^{-1} \Phi-z\left(1-z T_{1}\right)^{-1} \Phi=T_{2}^{*} \Phi N-T_{2}^{*}\left(1-z T_{1}\right)^{-1} \Phi(\Gamma z+N) .
\end{gathered}
$$

Since the characteristic function has the form $S(z)=K+\Psi\left(z-T_{1}\right)^{-1} \Phi(1.5)$, then after writing the expressions

$$
\begin{gathered}
S\left(\frac{1}{z}\right)-K=\Psi\left(\frac{1}{z}-T_{1}\right)^{-1} \Phi=z \Psi\left(1-z T_{1}\right)^{-1} \Phi, \\
T_{2} \Phi N+\Psi \widetilde{\sigma}_{2} K=0,
\end{gathered}
$$


we obtain the equality

$$
\begin{gathered}
\Psi^{*} \widetilde{\sigma}_{2}\left(S\left(\frac{1}{z}\right)-K\right)-z\left(1-z T_{1}\right)^{-1} \Phi=T_{2}^{*} \Phi N-T_{2}^{*}\left(1-z T_{1}\right)^{-1} \Phi(N+\Gamma z), \\
\Psi^{*} \widetilde{\sigma}_{2} S\left(\frac{1}{z}\right)-z\left(1-z T_{1}\right)^{-1} \Phi=-T_{2}^{*}\left(1-z T_{1}\right)^{-1} \Phi(N+\Gamma z), \\
T_{2}^{*}\left(1-z T_{1}\right)^{-1} \Phi=z\left(1-z T_{1}\right)^{-1} \Phi(N+z \Gamma)^{-1}-\Psi^{*} \widetilde{\sigma}_{2} S\left(\frac{1}{z}\right)(N+z \Gamma)^{-1}, \\
T_{2}^{*}\left(1-z T_{1}\right)^{-1} \Phi(1,1)=z\left(1-z T_{1}\right)^{-1} \Phi(N+z \Gamma)^{-1}(1,1)-\Psi^{*} \widetilde{\sigma}_{2} S\left(\frac{1}{z}\right)(N+z \Gamma)^{-1}(1,1) .
\end{gathered}
$$

Let us introduce the function $m(z)$ satisfying the equation

$$
(N+z \Gamma)^{-1}(1,1)=\frac{1}{m(z)}(1,1),
$$

i.e., suppose that $(1,1)$ is a latent vector of $(N+z \Gamma)$.

Then the statement

$$
T_{2}^{*} L_{x}(z)=\frac{L_{x}(z)}{m(z)}+\frac{\Psi^{*} \widetilde{\sigma}_{2} S\left(\frac{1}{z}\right)(1,1)}{m(z)}
$$

can be presented in the form

$$
\Psi^{*} \widetilde{\sigma}_{2} S\left(\frac{1}{z}\right)(1,1)=\widetilde{\mu} L_{x}(z)+\widetilde{\nu} \widetilde{L}_{x}(z),
$$

or by using the operator $\Psi^{*}$,

$$
(1,1) \sqrt{2} S\left(\frac{1}{z}\right) \widetilde{\sigma}_{2} \Phi_{x}=\widetilde{\mu} L_{x}(z)+\widetilde{\nu} \widetilde{L}_{x}(z) .
$$

Multiplying (3.15) by $L_{x}(z)$ and $\widetilde{L}_{x}(z)$, we obtain two statements

$$
\begin{aligned}
& \int_{0}^{l}(1,1) \sqrt{2} S\left(\frac{1}{z}\right) \widetilde{\sigma}_{2} \Phi_{t} d F_{t} L_{t}^{*}(\bar{z})=\widetilde{\mu}(z) \int_{0}^{l} L_{t}(z) d F_{t} L_{t}^{*}(\bar{z})+\widetilde{\nu} \int_{0}^{l} \widetilde{L}_{t}(z) d F_{t} L_{t}^{*}(\bar{z}), \\
& \int_{0}^{l}(1,1) \sqrt{2} S\left(\frac{1}{z}\right) \widetilde{\sigma}_{2} \Phi_{t} d F_{t} \widetilde{L}_{t}^{*}(\bar{z})=\widetilde{\mu}(z) \int_{0}^{l} L_{t}(z) d F_{t} \widetilde{L}_{t}^{*}(\bar{z})+\widetilde{\nu} \int_{0}^{l} \widetilde{L}_{t}(z) d F_{t} \widetilde{L}_{t}^{*}(\bar{z}) .
\end{aligned}
$$

By using previously obtained expressions for integrals (2.26)-(2.29), we introduce the following coefficients:

$$
d_{1}(z)=\int_{0}^{l} L_{t}(z) d F_{t} L_{t}^{*}(\bar{z})=\frac{E_{0}(z) \overline{E_{0}(\bar{z})}-\widetilde{E}_{0}(z) \overline{\widetilde{E}_{0}(\bar{z})}}{1-|z|^{2}},
$$




$$
\begin{gathered}
d_{2}(z)=\int_{0}^{l} \widetilde{L}_{t}(z) d F_{t} L_{t}^{*}(\bar{z})=\frac{G_{l}^{\prime}(z)+\widetilde{G}_{l}^{\prime}(z)}{2}, \\
d_{3}(z)=\int_{0}^{l} L_{t}(z) d F_{t} \widetilde{L}_{t}^{*}(\bar{z})=\frac{E_{0}^{\prime}(z)-\widetilde{E}_{0}^{\prime}(z)}{2}, \\
d_{4}(z)=\int_{0}^{l} \widetilde{L}_{t}(z) d F_{t} \widetilde{L}_{t}^{*}(\bar{z})=\frac{G_{l}(z) \overline{G_{l}(\bar{z})}-\widetilde{G}_{l}(z) \overline{G_{l}(\bar{z})}}{1-|z|^{2}} .
\end{gathered}
$$

Now, using the calculations from Lemma 5 , we obtain

$$
\begin{aligned}
& \int_{0}^{l} \Phi_{t} d F_{t} L_{t}^{*}(\bar{z})=\frac{1-z}{2 z}\left(\Phi_{l} J J\left(\begin{array}{c}
1 \\
1
\end{array}\right) \frac{1}{1-z}-I J \frac{1}{1-z}\left(\overline{E_{0}(\bar{z})}, \overline{\left.\widetilde{E}_{0}(\bar{z})\right)}\right),\right. \\
& \int_{0}^{l} \Phi_{x} a_{x} \widetilde{L}_{x}^{*}(\bar{z})=\frac{1-z}{2 z}\left(\Phi_{l} J \frac{1}{1-z}\left(\frac{\overline{G_{l}(\bar{z})}}{\widetilde{G}_{l}(\bar{z})}\right)-I J \frac{1}{1-z} I\left(\begin{array}{c}
1 \\
-1
\end{array}\right)\right) .
\end{aligned}
$$

By $I_{1}(z)$ and $I_{2}(z)$, we denote the following expressions:

$$
\begin{gathered}
I_{1}(z)=\frac{1}{2 z}(1,1) \sqrt{2} S\left(\frac{1}{z}\right) \widetilde{\sigma}_{2}\left(\Phi_{l}\left(\begin{array}{c}
1 \\
1
\end{array}\right)-J\left(\overline{E_{0}(\bar{z})}, \overline{\left.\widetilde{E}_{0}(\bar{z})\right)}\right),\right. \\
I_{2}(z)=\frac{1}{2 z}(1,1) \sqrt{2} S\left(\frac{1}{z}\right) \widetilde{\sigma}_{2}\left(\Phi_{l} J\left(\frac{\overline{G_{l}(\bar{z})}}{\widetilde{G}_{l}(\bar{z})}\right)+\left(\begin{array}{c}
1 \\
1
\end{array}\right)\right),
\end{gathered}
$$

then

$$
\begin{gathered}
\frac{I_{1}(z)}{d_{1}(z)}=\widetilde{\mu}(z)+\widetilde{\nu}(z) \frac{d_{2}(z)}{d_{1}(z)}, \\
\frac{I_{2}(z)}{d_{3}(z)}=\widetilde{\mu}(z)+\widetilde{\nu}(z) \frac{d_{4}(z)}{d_{3}(z)}, \\
\frac{I_{1}(z) d_{3}(z)-I_{2}(z) d_{1}(z)}{d_{1}(z) d_{3}(z)}=\nu(z) \frac{d_{2}(z) d_{3}(z)-d_{1}(z) d_{4}(z)}{d_{1}(z) d_{3}(z)} .
\end{gathered}
$$

Hence we have

$$
\nu(z)=\frac{I_{1}(z) d_{3}(z)-I_{2}(z) d_{1}(z)}{d_{2}(z) d_{3}(z)-d_{1}(z) d_{4}(z)}, \quad \widetilde{\nu}(z)=\frac{I_{1}(z) d_{4}(z)-I_{2}(z) d_{2}(z)}{d_{1}(z) d_{4}(z)-d_{2}(z) d_{3}(z)}
$$

and obtain the expression

$$
B_{L}\left(T_{2} f(z)\right)=\frac{F_{1}(z)}{m(z)}+\frac{\widetilde{\mu}(z)}{m(z)} F_{1}(z)+\frac{\widetilde{\nu}(z)}{m(z)} F_{2}(z),
$$

which proves the lemma. 
From Lemmas 6-11 we have the following theorem.

Theorem. Let a commutative knot $\triangle(1.21)$ be such that $E=\tilde{E}, \operatorname{dim} E=2$, $\sigma_{1}=\tilde{\sigma}_{1}=J_{N}(1.13)$, the spectrum of the operator $T_{1}$ be located at the point $\{1\}$ and the vector $(1,1)$ be latent for $(N+z \Gamma)$, i.e., let the function $m(z)$ be such that $(N+z \Gamma)(1,1)^{T}=m(z)(1,1)^{T}$, and the vector $(1,-1)$ be latent for $\widetilde{N}^{*}+z \widetilde{\Gamma}^{*}$, i.e., let the function $n(z)$ be such that $\left(\widetilde{N}^{*}+z \widetilde{\Gamma}^{*}\right)(1,-1)^{T}=n(z)(1,-1)^{T}$. Then the main system of the commutative operators $\left\{T_{1}, T_{2}\right\}$ of the knot $\triangle(1.21)$ is unitarily equivalent to the system of operators that operates in the de Branges space $\mathcal{B}(E, G)$ in the following way:

$$
\begin{gathered}
\left(T_{1} F\right)_{1}(z)=(z+\overline{\mu(\bar{z})}) F_{1}(z)+\nu(\bar{z}) F_{2}(z)+\frac{\overline{E_{0}(\bar{z})}-\overline{\widetilde{E}_{0}(\bar{z})}}{2} F_{2}(0), \\
\left(T_{1} F\right)_{2}(z)=\frac{F_{2}(z)-F_{2}(0)}{z}, \\
\left(T_{2} F\right)_{1}(z)=\frac{F_{1}(z)}{m(z)}+\frac{\widetilde{\mu}(z)}{m(z)} F_{1}(z)+\frac{\widetilde{\nu}(z)}{m(z)} F_{2}(z), \\
\left(T_{2} F\right)_{2}(z)=\frac{F_{2}(z) n(z)-F(0) n(0)}{z},
\end{gathered}
$$

where $\left(F_{1}(z), F_{2}(z)\right) \in \mathcal{B}(E, G)$. The coefficients $\mu(z), \nu(z)$ and $\widetilde{\mu}(z), \widetilde{\nu}(z)$ have the forms of (2.17), (2.18) and (3.7), (3.8), respectively, $N, \widetilde{N}, \Gamma, \widetilde{\Gamma}$ are defined by (1.22). The correctness of this definition follows from the reversibility of $\sigma$ and $\widetilde{\sigma}$.

Note. Let us consider the conditions $(N+z \Gamma)(1,1)^{T}=m(z)(1,1)^{T}$ and $\left(\widetilde{N}^{*}+z \widetilde{\Gamma}^{*}\right)(1,-1)^{T}=n(z)(1,-1)^{T}$ from the theorem. If we use $(1.22)$, then the condition of intertwining $[7]$

$$
S(z) N_{1}^{-1}\left(N_{2}+z \Gamma_{1}\right)=\widetilde{N}_{1}^{-1}\left(\widetilde{N}_{2}+z \widetilde{\Gamma}_{1}\right) S(z)
$$

will have the form

$$
S(z)(N+z \Gamma)=(\widetilde{N}+z \widetilde{\Gamma}) S(z) .
$$

After multiplying this equation from left by $(1,-1)$ and from right by $(1,1)^{T}$ and using $m(z)(1,1)^{T}=(N+z \Gamma)(1,1)^{T}$ and $(1,-1)(\widetilde{N}+z \widetilde{\Gamma})=(1,-1) \overline{n(\bar{z})}$, we obtain

$$
m(z)(1,-1) S(z)(1,1)^{T}=\overline{n(\bar{z})}(1,-1) S(z)(1,1)^{T} .
$$

Hence the conditions imply that either $m(z)=\overline{n(\bar{z})}$ or $(1,1) S(z)(1,1)^{T}=0$ for $\forall z \in C$.

Thus the functional model is built for the commutative system of the operators $T_{1}, T_{2}$, which is the main for the commutative knot $\triangle(1.21)$ satisfying the conditions of the theorem. However, $T_{1}$ and $T_{2}$ affect one of the components $\left[F_{1}(z), F_{2}(z)\right]$ as a shift and the other one as a multiplication by special holomorphic functions. 


\section{References}

[1] M.S. Livshits and A.A. Yantsevich, Theory of Operator Knots in Hilbert Spaces. Izdat. Kharkiv Univ., Kharkov, 1971. (Russian)

[2] V.A. Zolotarev, Analytical Methods of Spectral Representation of Non-unitary and Non-selfadjoint Operators. Kharkiv National University, Kharkov, 2003. (Russian)

[3] Lui De Branges, Hilbert Spaces of Entire Functions. Prentice-Hall, London, 1968.

[4] M.S. Livshits, About one Class of Linear Operators in Hilbert Space. - Math. Collection 19 (1946), No. 2, 236-260.

[5] B.S. Nagy and C. Foias, Harmonic Analysis of Operators in Hilbert Space. Mir, Moscow, 1970. (Russian)

[6] V.A. Zolotarev and V.N. Syrovatsky, De Branges Transform on the Cicle. Vestnik KNU V.N. Karazin. Number 711, Series "Mathematics and Applied Mechanics", Kharkiv National University, Kharkiv, 2005. (Russian)

[7] V.A. Zolotarev, Model Representations of Commutative Systems of Linear Operators. - Funct. Anal. and Appl. 22 (1988), No. 1, 66-68.

[8] V.A. Zolotarev, Functional Model of Commutative Operator Systems. - J. Math. Phys., Anal., Geom. 4 (2008), No. 3, 420-440.

[9] V.N. Syrovatsky, Functional Models of Commutative Systems of Operators Close to the Unitary. Vestnik KNU V.N. Karazin. Number 1018, Series "Mathematics and Applied Mechanics", Kharkiv National University, Kharkiv, 2012. (Russian) 